\title{
Terapia de reposição hormonal na menopausa
}

\author{
Hormone replacement therapy in menopause
}

Dolores Pardini'

\section{RESUMO}

Embora o estrógeno já esteja disponível para venda há mais de seis décadas, as mulheres ainda permanecem confusas quanto ao risco e aos benefícios da terapia hormonal na menopausa (THM), terapia estrogênica isolada ou associada a progestágenos. A publicação de estudos controlados,

${ }^{1}$ Ambulatório de Menopausa, Disciplina de Endocrinologia, Universidade Federal de São Paulo, Escola Paulista de Medicina (Unifesp/EPM). Departamento de Endocrinologia Feminina e Andrologia, Sociedade Brasileira de Endocrinologia e Metabologia (SBEM). Disciplina de Endocrinologia, Departamento de Medicina, Unifesp/EPM, São Paulo, SP, Brasil

\author{
Correspondência para: \\ Dolores Pardini \\ Rua Júpiter, 160, ap. 111 \\ 01532-030 - São Paulo, SP, Brasil \\ dpardini@uol.com.br \\ Recebido em 2/Out/2013 \\ Aceito em $27 / \mathrm{Nov} / 2013$
} randomizados, como o Heart and Estrogen/progestin Replacement Study (HERS) e Women's Health Initiative (WHI), intensificou essa controvérsia risco/benefício. Milhares de mulheres são tratadas com THM para alívio dos sintomas menopausais, incluindo sintomas vasomotores e sudorese, principal indicação da estrogenoterapia. Outras podem persistir no tratamento na esperança de prevenir doenças crônicas. A manutenção da massa óssea e a prevenção de fraturas são efeitos do estrógeno já bem estabelecidos. Estudos observacionais dos efeitos metabólicos e vasculares do estrógeno sugerem um benefício em potencial na redução do risco de doenças vasculares, mas estudos randomizados e controlados não demonstraram nenhuma evidência de que a terapia hormonal pudesse beneficiar as mulheres com doença vascular previamente instalada ou em mulheres aparentemente saudáveis. $\mathrm{O}$ aumento do risco de câncer de mama e doença tromboembólica tem se confirmado nesses estudos. A incidência em números absolutos de efeitos adversos é baixa e o risco individual no primeiro ano de tratamento é muito baixo. Os riscos são cumulativos com o tempo de uso. A relação risco/benefício deve ser individualizada. Arq Bras Endocrinol Metab. 2014;58(2):172-81

\section{Descritores}

Menopausa; terapia de reposição; estrógeno; efeitos colaterais; janela de oportunidade

\begin{abstract}
Although estrogen has been clinically available for more than six decades, women have been confused by different opinions regarding the risks and benefits of menopausal hormone therapy (HT), estrogen therapy (ET), and estrogen-progestin therapy (EPT). The publication of randomized controlled trials (RCTs), notably, the Heart and Estrogen/progestin Replacement Study (HERS) and Women's Health Initiative (WHI), has intensified the risk vs. benefit controversy. Millions of women are treated with HT for relief of menopausal symptoms, including vasomotor flushes and sweats, for which estrogen is uniquely and highly effective. Others may continue longer-term treatment in the hope that HT will help to prevent chronic disease. The preservation of bone mass with continuing estrogen therapy and reduction of subsequent risk of fracture is well established. Observational studies of the metabolic and vascular effects of estrogens have suggested a potential benefit in reducing the risk of vascular disease, but recently published randomized controlled trials demonstrated no evidence of benefit in women with established vascular disease or in apparently healthy women. The increased risks of breast cancer and thromboembolic disease have been confirmed in these trials, with evidence of increased risk of stroke. The absolute incidence of an adverse event is low, and the risk of stroke in an individual woman in a single year is very small, but with long-term use, the risks are cumulative over time. The risk-benefit balance needs to be individualized for each woman. Arq Bras Endocrinol Metab. 2014;58(2):172-81
\end{abstract}

Keywords

Menopause; replacement therapy; estrogen; side effects; windows safe

\section{INTRODUÇÃO}

A terapia de reposição hormonal na menopausa Х (THM) tem sido objeto de muita discussão e especulação desde a década de 1960, período no qual se prescrevia estrogenoterapia isolada para todas as mulheres menopausadas, dando origem a complicações principalmente em nível endometrial (1). Em 1980, após o efeito protetor das progestinas (compostos que 
interagem com os receptores da progesterona nos tecidos alvo com efeito similar à progesterona) no endométrio ser estabelecido, a THM obteve outra ascensão. Na década de 1990, a THM atingia seu apogeu quando os estudos em animais e os observacionais sugeriam que a estrogenoterapia pós-menopausa podia prevenir a doença coronariana e a demência, além de evitar a perda de massa óssea. Antes de 2002, não se questionavam os benefícios da THM quanto à melhora dos sintomas menopausais associada a uma redução do risco cardiovascular, osteoporose e câncer de cólon. Os riscos de câncer de mama e tromboembolismo eram compensados pelas vantagens principalmente com base em estudos observacionais. Por volta do século 21 , aproximadamente 15 milhões de mulheres americanas faziam uso de reposição hormonal na menopausa, diminuindo substancialmente após 2002 (1). Dados oriundos do NHANES (2009-2010) sugerem o uso de THM em $4,7 \%$ de mulheres acima de 40 anos e de 6,7\% nas mulheres entre 50 e 59 anos de idade, comparado com 38,3\% de usuárias entre 1999 e 2000 (2). A publicação do estudo Heart and Estrogen/progestin Replacement Study (HERS) (3) em 1998 seguida pelos resultados do Women's Health Initiative (WHI) (4) em 2002 e, posteriormente, em 2004 (5) marcou outra fase da THM, na qual se estabeleceram alguns critérios para tratamento da menopausa, tais como idade da paciente, tempo de menopausa, sintomas, doses, vias de administração, comorbidades etc. Surgia o conceito individualização. Essas publicações geraram 10 anos de controvérsia na análise detalhada de seus resultados. A Endocrine Society Scientific Statements, ao rever toda a literatura publicada sobre THM, valendo-se apenas dos ensaios clínicos com metodologia controlada e classificando todas as conclusões com o método GRADE ( Grading of Recommendations Assessment, Development and Evaluation) (6), publicou um posicionamento rigorosamente documentado e com todas as conclusões a respeito dos riscos e benefícios da THM classificadas de acordo com o grau de evidência (7). As conclusões estão divididas naquelas que permaneceram inalteradas com o passar do tempo, com base em estudos experimentais de melhor consistência (grau de evidência A), aquelas que permaneceram inalteradas, porém com um nível menor de certeza, baseadas em estudos experimentais ou observacionais de menor consistência (grau de evidência B), e aquelas baseadas em relatos de caso e estudos não controlados (grau de evidência C, D). As recomenda- ções relatadas a seguir expressam um resumo da literatura em relação aos princípios da terapia hormonal, numa visão simples e global, que servem como plataforma comum sobre questões relacionadas aos vários aspectos do tratamento hormonal.

\section{RISCOS E BENEFÍCIOS DA THM}

\section{Conclusões com grau de evidência A}

\section{Sintomas vasomotores}

Os fogachos são os sintomas menopausais mais frequentes, afetando $60 \%$ a $80 \%$ das mulheres. Para as portadoras de fogachos e/ou sudorese noturna, uma revisão sistemática do Instituto Cochrane calculou uma redução de $75 \%$ na frequência e $87 \%$ na severidade dos sintomas vasomotores nas usuárias de hormonioterapia (8). A maioria dos dados publicados sobre o uso de THM e fogachos é baseada em doses "standart" de estrógeno (estrógeno conjugado $0,625 \mathrm{mg} ; 17-\beta$ estradiol oral $1 \mathrm{mg}, 17-\beta$ estradiol transdérmico $50 \mu \mathrm{g} / \mathrm{d}$ ), entretanto, baixas doses de estrógeno também são efetivas para alívio dos sintomas vasomotores. Alternativas não hormonais para os fogachos incluem antidepressivos e gabapentina. Embora esses agentes não sejam tão efetivos como o estrógeno, são significantemente superiores ao placebo $(9)$.

\section{Sintomas urogenitais}

Os sintomas urogenitais incluem: bexiga hiperativa (BHA), incontinência urinária (IU), infecção recorrente do trato urinário (IRTU) e atrofia vaginal. A BHA afeta mais de 50 milhões de pessoas dos países em desenvolvimento acompanhada ou não de incontinência. Todas as metanálises e revisões encontraram melhora dos sintomas urogenitais com a estrogenoterapia local ou sistêmica. A revisão sistemática do Cochrane concluiu que o estrógeno também melhora a incontinência (10). Doses baixas de estrógeno local normalizam a atrofia vaginal e reduzem a incidência de infecção urinária recorrente. Quando o estrógeno em baixas doses é administrado somente na vagina, não se faz necessária a associação de progesterona para proteção endometrial nas mulheres com útero. Ainda não existem dados suficientes que confirmem a segurança da estrogenoterapia local nas mulheres com antecedentes de câncer de mama. A terapia não hormonal com hidratantes deve ser considerada nesses casos (11). 


\section{Osteoporose pós-menopausa}

O estrógeno isolado ou associado à progesterona é eficaz na prevenção da perda óssea associada à menopausa e na redução da incidência de fratura vertebral e não vertebral, incluindo pacientes de baixo risco. Embora a magnitude do declínio na renovação óssea esteja relacionada aos níveis de estrogênio, a reposição em baixas doses também tem influência positiva na massa óssea da maioria das mulheres. Com base nas evidências, a THM é a terapia de primeira linha para mulheres pós-menopausadas que apresentam alto risco de fratura e estejam abaixo dos 60 anos de idade, na presença ou não de sintomas menopausais com a mesma efetividade dos bisfosfonatos $(1,12)$. O efeito protetor da THM sobre a densidade mineral óssea diminui após suspensão do hormônio. Iniciar a THM com dose padrão não é recomendado para fins exclusivos de prevenção de fratura após os 60 anos de idade. O raloxifeno, modulador seletivo do receptor de estrógeno, aumenta a massa óssea e reduz a incidência de fratura vertebral, mas não reduz a fratura de quadril.

\section{Câncer de cólon}

A reposição estroprogestativa reduz o risco de câncer de cólon. O modo de ação ainda permanece desconhecido, embora várias observações demonstrem que o tecido colônico é hormonalmente influenciado. O estrógeno decresce a concentração de ácidos biliares, os quais se acredita promoverem alterações malignas no cólon. Hipotetiza-se que os progestágenos atuem com efeito antiproliferativo no ciclo proteico das células colônicas. Na prática clínica, a THM não deve ser considerada para prevenção do câncer colorretal, mas não deve ser excluída naquelas mulheres sintomáticas com alto risco para a patologia (1).

\section{Tromboembolismo venoso}

A THM aumenta o risco de fenômenos tromboembólicos em duas vezes aproximadamente, risco esse incrementado pela obesidade, trombofilia, idade superior a 60 anos, cirurgia e imobilização (13). A via de administração do estrógeno, a dosagem e o tipo de progestágeno associado ao estrógeno podem afetar o risco do evento tromboembólico. A terapia combinada com estrógeno mais progesterona aumenta o risco de tromboembolismo quando comparado com a monoterapia estrogênica. Estudos observacionais sugerem que a progesterona micronizada ou didrogesterona tem menor risco do que outros progestágenos e esse risco é maior durante o primeiro ano de reposição. A reposição estrogênica por via transdérmica tem se mostrado mais segura quanto aos fenômenos tromboembólicos que a via oral (1).

\section{Endométrio}

A administração de estrógeno sem oposição induz a estímulo do endométrio, aumentando risco de câncer e hiperplasia endometrial. A associação estroprogestativa confere proteção endometrial. As mulheres com útero devem receber a associação com prostágeno por, no mínimo, 12 dias por mês nos esquemas sequenciais. Por outro lado, os esquemas combinados contínuos conferem uma proteção endometrial maior quando comparados aos esquemas cíclicos (1).

\section{Acidente vascular cerebral}

$\mathrm{O}$ risco de acidente vascular cerebral (AVC) aumenta exponencialmente com o avançar da idade. A THM pode ser responsável por 1 caso adicional em 10.000 mulheres que iniciaram o tratamento antes dos 50 anos de idade, 2 casos para mulheres entre 55 e 60 anos e 7 casos para mulheres com idade superior a 65 anos. A THM não reduz a incidência de AVC em mulheres idosas com doença vascular preexistente. O risco de AVC, além da idade, também pode ser dependente da dose, da via de administração do estrógeno e da associação com progestágenos. A via não oral em alguns estudos está associada a um menor risco de $\operatorname{AVC}(13,14)$.

\section{Cognição}

Diversos estudos observacionais e metanálises sugerem que se o estrógeno for prescrito na mulher jovem em perimenopausa acarreta uma diminuição do risco de doença de Alzheimer ou retarda seu aparecimento. Acredita-se que o "timing" do início da reposição é crítico nesses casos à semelhança dos eventos cardiovasculares. THM iniciada após os 60 anos de idade não melhora a memória (14). O estrógeno parece possuir diferentes efeitos no cérebro dependentes da idade da paciente, idade no início da reposição, tipo de menopausa (natural ou induzida) e tipo de reposição utilizada (1). A THM não está indicada para a prevenção primária ou secundária de demência.

\section{Mama}

A incidência de câncer de mama varia de acordo com os diferentes países. Assim, os dados atualmente disponíveis não podem ser obrigatoriamente generalizados. 
O grau de associação entre o câncer de mama e a THM continua controverso. Entretanto, o único dado considerado com grau de evidência A é que a administração de estrógeno isolado ou associado à progesterona aumenta a percentagem de densidade mamária (PMD) e o raloxifeno diminui o risco de câncer de mama. O "International Breast Cancer Intervention Study" (IBIS) mostrou uma redução de $13,7 \%$ na PMD com o uso de tamoxifeno comparado a $7,3 \%$ no grupo placebo durante quatro anos e meio de seguimento (15). Nas mulheres que apresentaram uma redução de $10 \%$ ou mais da PMD, o risco de câncer de mama foi reduzido em $52 \%$ comparado ao grupo placebo $(\mathrm{p}<0,01)$. A PDM é um forte fator de risco para a neoplasia de mama, sendo influenciada por algumas formas de THM. A associação de estrógeno e progesterona aumenta a PMD em 3\% a $5 \%$, significantemente maior que o placebo e estrógeno isoladamente. No estudo controlado WHI, não foi observado nenhum aumento do risco de câncer de mama em mulheres que fizeram uso de estrógeno isolado por até sete anos, levando-se em conta que a maioria das participantes do estudo WHI possuía sobrepeso ou obesidade (4). Dados do WHI sugerem que a administração exclusiva de estrógeno por longo período, por sete e quinze anos respectivamente, não aumentou $o$ risco de câncer de mama em mulheres americanas (5). Uma observação bastante atual é que o uso de estrógeno isolado por menos de cinco anos pode reduzir o risco de câncer de mama em pacientes que iniciaram a reposição muitos anos após a menopausa, fenômeno denominado "gap time". Dados oriundos do estudo SEER (Surveillance, Epidemiology and End Results) mostraram que mulheres com idade entre 50 e 54 anos tinham 13/1.000 de chance de desenvolver câncer de mama após cinco anos de menopausa, enquanto aquelas que iniciaram a estrogenoterapia após cinco anos apresentaram risco de 2,59/1.000 (7). Uma possível explicação para o fato seria o estrógeno induzindo apoptose. As células cancerígenas da mama em deprivação estrogênica por longo tempo em meio de cultura (mimetizando o "gap time") adaptariam-se e tornariam-se sensíveis aos efeitos pró-apoptóticos do estradiol. Em mulheres, esse efeito pró-apoptótico paradoxal poderia encolher o tamanho de tumores ocultos já existentes e reduzir a taxa de detecção clínica tardia dos cânceres. Não existem dados suficientes para avaliar as possíveis diferenças na incidência do câncer de mama usando diferentes tipos e vias de administração de estrogênio, progesterona natural, progestógenos e androgênios.

\section{Conclusões com grau de evidência B}

\section{Diabetes e intolerância a hidratos de carbono}

$\mathrm{O}$ uso de estrógeno isolado ou combinado à progesterona conforme observado no estudo WHI e no estudo HERS está associado à diminuição no risco de diabetes melito tipo II (DMII) e menor acúmulo de tecido gorduroso abdominal e/ou periférico. O risco de desenvolver DMII aumenta com a maturidade da mulher, evidentemente associado a outros fatores, como obesidade central e sedentarismo. O declínio da produção de estrógeno pelos ovários na menopausa também pode ter um papel importante, entretanto, essa possibilidade ainda não está completamente estabelecida e a literatura permanece controversa. Os efeitos da THM no metabolismo dos hidratos de carbono podem ser diretos, isto é, no pâncreas ou na musculatura esquelética, melhorando a sensibilidade à insulina, ou indiretos, reduzindo o acúmulo de gordura visceral. Esses efeitos dependem da via de administração, da dose e do tipo do estrogênio utilizado na reposição pós-menopausa $(7,15)$.

\section{Doença cardiovascular}

A doença cardiovascular é a principal causa de morbidade e mortalidade em mulheres na pós-menopausa. A menopausa pode ser considerada fator de risco para a doença arterial coronária em mulheres devido a efeitos potenciais da senescência ovariana sobre a função cardíaca, pressão arterial e alguns parâmetros metabólicos, como tolerância à glicose e perfil lipídico. Os modelos animais, a ciência básica e os estudos observacionais sustentam a hipótese de que a THM pode prevenir a aterosclerose e reduzir os eventos cardiovasculares (ECV) por meio de efeitos sistêmicos ou genômicos e não genômicos diretamente no coração e nos vasos. As diversas análises posteriores aos resultados observados no estudo WHI concluíram que o desfecho negativo deveu-se a idade das pacientes ser muito avançada (média de 63 anos) no início da terapia, altas doses de estrógeno para a faixa etária, via de administração e tempo decorrido desde a última menstruação. Combinando os dados oriundos de vários estudos observacionais e randomizados no consenso do NAMS, concluiu-se que as mulheres que iniciam a THM após 10 anos de menopausa apresentam risco aumentado de ECV e aquelas que iniciam antes desse período tendem a ter baixo risco de ECV, fato conhecido como janela 
de oportunidade (14). O consenso da sociedade internacional de menopausa corroborou a conclusão acima e acrescentou que a THM não está contraindicada a mulheres hipertensas e, em alguns casos, a THM pode reduzir a pressão arterial. Por outro lado, a THM está contraindicada a mulheres com história de infarto do miocárdio, acidente vascular cerebral e embolia pulmonar (16). O estudo Danish Osteoporosis Prevention Study (DOPS) (17), randomizado, envolvendo 1.006 mulheres seguidas por 10 anos, muito contribuiu para reforçar o conceito de janela de oportunidade e os benefícios da THM nos eventos cardiovasculares. Após 10 anos de seguimento, as mulheres jovens que foram tratadas na perimenopausa apresentaram resultados semelhantes aos estudos observacionais, ou seja, uma redução significante de eventos cardiovasculares e mortalidade sem aparente aumento de câncer, tromboembolismo venoso ou AVC, comparadas ao grupo controle. Acredita-se que a administração oral do estrógeno aumentaria os fatores pró-inflamatórios, tais como a matriz metaloprotease 9, a qual, agindo na placa ateromatosa, acarretaria instabilidade e ruptura desta, gerando eventos tromboembólicos (14). Na mulher jovem, embora ocorra esse efeito, não encontra substrato aterosclerótico para agir. A continuação da THM além dos 60 anos de idade, uma vez iniciada na perimenopausa, deve ser decidida como parte da análise geral da relação risco/ benefício.

\section{Qualidade de vida}

A transição menopausal e os anos subsequentes podem estar associados a um declínio considerável na qualidade de vida decorrente da diminuição dos níveis de estrógeno circulantes. A THM acarreta melhora da qualidade de vida à medida que diminuem os sintomas vasomotores, a insônia e a labilidade de humor nas mulheres sintomáticas. Quando a reposição é iniciada logo após a menopausa cirúrgica, pode haver benefícios na memória verbal (1).

\section{Ganho de peso}

O receio de ganhar peso com a reposição hormonal constitui uma das maiores causas de má aderência e abandono da THM, entretanto a maioria dos estudos mostra o contrário, as usuárias ganham menos peso e gordura corporal que as não usuárias. O Instituto $\mathrm{Co}^{-}$ chrane, em revisão sistemática em 2002 e atualizada em 2010 envolvendo 90 estudos, concluiu que não existem evidências de que a THM com estrógeno isolado ou combinado com progestágeno acarrete modificação no peso corporal, indicando que esses regimes não causam ganho extra de peso em adição ao ganho observado na menopausa $(18,19)$.

\section{TH E MORTALIDADE}

Embora seja consenso o aumento da ocorrência dos fenômenos tromboembólicos e da incidência do câncer de mama nas usuárias da reposição estrogênica conforme as considerações anteriormente descritas, também é consensual que a estrogenoterapia iniciada em perimenopausa, no grupo alvo de mulheres entre 50 e 59 anos, está associada a uma redução de $40 \%$ na mortalidade. Previamente aos estudos HERS e WHI, estudos epidemiológicos já publicaram redução na mortalidade em mulheres sob estrogenoterapia comparadas com menopausadas sem reposição. Entretanto, a melhor evidência do efeito da THM na mortalidade é proveniente de uma metanálise que reuniu 19 estudos randomizados, controlados, duplo-cegos envolvendo 16.000 mulheres com idade média de 55 anos. Observou-se uma redução da mortalidade em termos absolutos de 84\% nas pacientes tratadas, isto é, uma em cada 119 mulheres tratadas com terapia hormonal em cinco anos não morreu, em comparação com as não tratadas (20).

\section{VIA DE ADMINISTRAÇÃO}

O estradiol e o progestógeno, quando administrados por via não oral, impedem o metabolismo de primeira passagem pelo fígado, ocasionando, assim, menor potencial para estímulo das proteínas hepáticas, fatores de coagulação e perfil metabólico neutro, o que pode ser mais favorável em termos de risco cardiovascular e fenômenos tromboembólicos. O risco de tromboembolismo venoso se mostrou menor quando usado o estradiol por via transdérmica comparado com o estradiol por via oral. Entretanto, não foi confirmado se isso se deveu ao impacto diferencial do estradiol sobre os fatores de coagulação sintetizados no fígado. Por outro lado, a administração por via oral acarreta um maior impacto na redução dos níveis do colesterol LDL, o que é uma vantagem na mulher com hipercolesterolemia e triglicérides normais, levando-se em conta que o último pode elevar-se com a medicação. A primeira passagem uterina da administração vaginal de progestagênios acarreta concentrações locais adequadas e boa 
proteção endometrial com níveis sistêmicos do progestágeno menores. A combinação do uso não oral do estradiol combinado ao progestágeno intrauterino pode melhorar a aderência e minimizar os riscos da THM. Entretanto, ainda são necessários estudos de boa qualidade para confirmar essa hipótese. Uso vaginal de estradiol é preferencial no tratamento isolado das queixas urogenitais (15) (Tabela 1).

Tabela 1. Vias de administração de estrógeno

\begin{tabular}{lll}
\hline & Via oral & Via não oral \\
\hline Vantagens & Mais difundida & Relação $\mathrm{E}_{2} / \mathrm{E}_{1}>1$ \\
& Custo menor & $\downarrow$ Triglicérides \\
& Menos alérgica & Evita os efeitos decorrentes da \\
& $\uparrow$ HDL; $\downarrow$ LDL & passagem hepática \\
Desvantagens & Angiotensinogênio & Custo maior \\
& $\uparrow$ SHBG, TBG, CBG & Alergia cutânea local 2-24\% \\
& $\uparrow$ Triglicérides & Impacto discreto no HDL e LDL \\
& Relação $\mathrm{E}_{2} / \mathrm{E}_{1}<1$ & \\
& $\downarrow$ Antitrombina III & \\
\hline
\end{tabular}

Fonte: Pardini (15).

\section{CLASSIFICAÇÃO DOS ESTRÓGENOS}

\section{Sintéticos}

Os principais são o etinilestradiol, mestranol, quinestrol e dietilestilbestrol. Devido ao fato de não serem oxidados pela desidrogenase que oxida o 17- $\beta$ estradiol, seu efeito no fígado é acentuado, levando-o à produção de proteínas como SHBG, substrato de renina e outras, às vezes indesejáveis, independentemente da via de administração. Por esse motivo, embora exerça efeito no osso, seu uso é restrito aos anticoncepcionais orais.

\section{Naturais}

Os mais frequentemente utilizados na TRH são os estrogênios conjugados e o estradiol transdérmico ou percutâneo, seguidos pelo valerianato de estradiol e o estradiol micronizado. Quando administrados por via oral, todos resultam em níveis mais elevados de estrona e seus conjugados do que de estradiol, sendo que essa transformação se processa na mucosa gastrintestinal e no fígado. $\mathrm{O}$ estriol, apesar de provocar poucos efeitos colaterais, não previne a perda de massa óssea. Tanto os estrogênios sintéticos como os naturais têm se mostrado úteis na preservação da massa óssea e na melhora da sintomatologia, entretanto na terapia de reposição hormonal do climatério e menopausa os naturais estão mais indicados (Tabela 2).

\section{Dose de estradiol}

A melhor dose é a menor dose efetiva para cada mulher. Baixas doses de estradiol isoladamente ou estradiol associado ao progestágeno são mais bem toleradas e podem apresentar uma relação custo/benefício melhor que a dose padrão. Entretanto, doses baixas e principalmente as ultrabaixas ainda não têm o suporte de estudos controlados e prospectivos de boa qualidade (7) (Tabela 2).

Tabela 2. Classificação e apresentação dos estrógenos usados em THM

\begin{tabular}{lc}
\hline Estrógeno & Apresentação \\
\hline I - Oral & \\
la - Derivados da estrona & \\
Estrógenos equinos conjugados & 0,$3 ; 0,625 ; 1,25 ; 2,5 \mathrm{mg}$ \\
Ib - Derivados do estradiol & \\
Valerianato de estradiol & $1-2 \mathrm{mg}$ \\
Estradiol micronizado & $1-2 \mathrm{mg}$ \\
Estriol & $1-2 \mathrm{mg}$ \\
IC - Sintéticos & \\
Etinil estradiol & 0,$02 ; 0,05 ; 0,5 \mathrm{mg}$ \\
II - Injetável & \\
Benzoato de estradiol & $0,5 \mathrm{mg} / \mathrm{mL}$ \\
Fosfato de poliestradiol & $40 \mathrm{mg} / \mathrm{mL}$ \\
Estrógenos equinos conjugados & $25 \mathrm{mg} / \mathrm{mL}$ \\
Valerianato de estradiol & $10 ; 20 ; 40 \mathrm{mg} / \mathrm{mL}$ \\
III - Vaginal & \\
Estrógenos equinos conjugados & $0,625 \mathrm{mg} / \mathrm{dose}$ \\
Estriol & \\
IV - Implante & \\
Estradiol & \\
V - Transdérmico e percutâneo & $25 ; 50 ; 100 \mathrm{mg} / \mathrm{pellet}$ \\
Estradiol TTS & \\
Estradiol gel & \\
\hline
\end{tabular}

Fonte: Pardini (15).

\section{Progestagênios}

A associação do progestágeno ao estrógeno é obrigatória em pacientes com útero intacto ou em pacientes com histerectomia parcial em que existe resíduo de cavidade endometrial. A indicação primária da adição do progestágeno à estrogenoterapia refere-se à proteção endometrial contra a hiperplasia e o adenocarcinoma associados à reposição isolada de estrógeno. Não está recomendada quando baixas doses de estrógeno são administradas por via vaginal no tratamento da atrofia vaginal isoladamente. Os dados atuais de 
que a associação do progestágeno aumenta o risco de câncer de mama são ainda inconsistentes e controversos, mas está bem estabelecido que a adição de progesterona à terapia hormonal não diminui o risco de câncer de mama. Entretanto, existe a preocupação de que a adição do progestágeno não prejudique ou atenue os efeitos benéficos do estrógeno, principalmente no sistema cardiovascular e na massa óssea, bem como sobre um bem-estar da paciente usuária da THM. Ainda que seja obrigatória a promoção de atividade secretória em endométrio previamente estrogenizado para que uma determinada substância possa ser caracterizada como progestagênio, as ações específicas sobre outros órgãos e tecidos diferem substancialmente entre os diferentes tipos de progestágenos empregados na THM. Eles podem ser derivados da molécula de progesterona, testosterona ou espironolactona e, devido suas diferentes origens, possuem maior ou menor afinidade com os receptores de progesterona, testosterona, estradiol e aldosterona (Tabela 4). Várias moléculas novas de progestágenos foram sintetizadas nas últimas duas décadas, sendo consideradas de quarta geração. Incluem-se, nessa geração, a drospirenona, trimegestona, nesterona e acetato de nomegestrol. A nesterona não está disponível para THM no mercado brasileiro. A segurança e os benefícios da escolha do progestágeno à semelhança da escolha do estrogênio baseiam-se na individualização. A drosperinona, por ser um esteroide essencialmente com atividade antimineralocorticoide, está mais indicada a pacientes com predisposição à retenção hídrica. Por possuir efeito antiandrogênico, também pode ser uma boa escolha nas mulheres hirsutas ou hiperandrogênicas. Não existe consenso a respeito da melhor via de administração do progestágeno, bem como do tipo ou da dose ideal a ser utilizada para minimizar os efeitos colaterais sem comprometer a proteção endometrial (21) (Tabela 5).

Tabela 3. Doses de estrógeno usadas em terapia hormonal da menopausa nos Estados Unidos e outros países

\begin{tabular}{|c|c|c|c|c|}
\hline $\begin{array}{l}\text { Estados } \\
\text { Unidos }\end{array}$ & $\begin{array}{l}\text { Outros } \\
\text { países }\end{array}$ & $\begin{array}{l}\text { Estradiol } \\
\text { (mg) via } \\
\text { oral }\end{array}$ & $\begin{array}{l}\text { Estrógeno } \\
\text { conjugado } \\
\text { (mg) via oral }\end{array}$ & $\begin{array}{l}\text { Estradiol } \\
\text { (mg) via } \\
\text { transdérmica }\end{array}$ \\
\hline Alta & Padrão & 2,0 & 1,25 & 0,10 \\
\hline Padrão & Baixa & 1,0 & 0,625 & 0,05 \\
\hline Baixa & Ultrabaixa & 0,5 & $0,3-0,45$ & 0,025 \\
\hline Ultrabaixa & Microdose & 0,25 & - & 0,014 \\
\hline
\end{tabular}

Adaptada de: Birkhäuser (27).
Tabela 4. Afinidade dos diferentes progestágenos aos diferentes receptores esteroides

\begin{tabular}{lcccccc}
\hline & Prog & Andro & Antiandro & Gluco & Antimineral & Estro \\
\hline Progesterona & + & - & + & - & + & - \\
NETA & ++ & + & - & - & - & $+/-$ \\
LNG & +++ & ++ & - & - & $+/-$ & - \\
MPA & +++ & + & - & + & - & - \\
TMG & ++++ & - & + & - & ++ & - \\
\hline
\end{tabular}

Prog: atividade progestacional; Andro: atividade androgênica; Antiandro: atividade antiandrogênica; Gluco: atividade glicocorticoide; Antimineral: atividade antimineralocorticoide; Estro: atividade estrogênica; NETA: acetato de noretindrona; LNG: levonorgestrel; MPA: acetato de medroxiprogesterona; TMG: trimegestona.

Tabela 5. Características específicas das novas moléculas de progestágenos*

\begin{tabular}{ll}
\hline Progestágeno & Principal efeito \\
\hline Dienogest & Antiandrogênico \\
Drosperinona & Antimineralocorticoide \\
Nestorona & Altamente progestacional e antigonadotrófica \\
Ac. nomegestrol & Altamente antigonadotrófica \\
Trimegestona & Altamente progestacional \\
\hline
\end{tabular}

* Nenhum possui ação androgênica ou estrogênica.

\section{Esquemas terapêuticos}

São inúmeras as formas de administrar a THM, visando ao alívio dos sintomas e, acima de tudo, à proteção endometrial quando associamos a progesterona ao estrógeno. Os consensos atuais recomendam minimizar a exposição ao progestágeno. Os esquemas combinados podem ser cíclicos ou contínuos. No primeiro o estrógeno é dado de forma contínua e o progestágeno é dado 10-12 dias por mês e, no segundo, ambos são administrados conjuntamente de forma ininterrupta. No esquema cíclico, a mulher apresenta sangramento ao final de cada ciclo de progesterona e, no contínuo, a grande maioria entra em amenorreia. Quando isso não ocorre, devemos investigar as condições do endométrio. De qualquer forma, a escolha do esquema é sempre individualizada, priorizando-se a vontade da paciente e o tempo de menopausa.

\section{Reposição androgênica}

O último posicionamento da Sociedade de Endocrinologia Americana sustenta que o diagnóstico de insuficiência androgênica na mulher não está bem definido (22). O Consenso de Princeton, em 2002, sugeria um quadro clínico de falta de motivação, fadiga, mal-estar, humor depressivo, disfunção sexual, diminuição de pelos pubianos e de massa muscular, síndrome climatérica e perda óssea não responsivas a estrogênio (23). La- 
boratorialmente, a testosterona total estaria $<150 \mathrm{pg} /$ $\mathrm{mL}$, a testosterona livre por diálise de equilíbrio $<1 \%(2$ $\mathrm{pg} / \mathrm{mL}$ ) ou S-DHEA $<100 \mathrm{ng} / \mathrm{mL}$, devendo as dosagens de testosterona ser colhidas pela manhã e no meio do ciclo em mulheres na pré-menopausa. O próprio Consenso, porém, admitia que os kits para dosagens de androgênios são inapropriados para valores baixos. Em mulheres esses níveis estão frequentemente abaixo da sensibilidade do ensaio, portanto tratava-se de um quadro clínico inespecífico de comprovação laboratorial difícil. Tanto os androgênios ovarianos quanto os adrenais sofrem redução em mulheres a partir dos 25 anos $(24,25)$, principalmente no início dos anos reprodutivos. A queda é contínua com a idade e mais precoce e acentuada nos androgênios adrenais. Entre mulheres de 45 a 54 anos com menopausa natural, o ovário continua a secretar androgênios. Como a queda de estrogênios é da ordem de 16 vezes e a de androgênios de apenas duas a quatro vezes, ocorre um hiperandrogenismo relativo na menopausa, mesmo com níveis absolutos baixos de androgênios. Já em mulheres com ooforectomia bilateral os níveis de testosterona total e livre calculada caem significativamente (24). Outros grupos de mulheres de risco para insuficiência androgênica são as com falência ovariana prematura, as em tratamento com antiandrogênios, contraceptivos orais ou terapia hormonal por via oral (que reduzem o LH e aumentam a SHBG, diminuindo os androgênios livres), as com insuficiência adrenal primária ou secundária ao uso de corticoides ou a hipopituitarismo. As indicações clássicas e previamente estabelecidas para prescrevermos testosterona na mulher são na esfera sexual como diminuição da libido e prazer sexual. Efeitos como ganho de massa óssea e aumento da massa muscular também são bem estabelecidos com o uso de testosterona (12). Mais recentemente diversos estudos correlacionaram a testosterona com proliferação celular na mama e o câncer de mama. Já existem números considerados de evidências de que tanto a testosterona como seu derivado deidrotestosterona exercem efeito inibitório no crescimento da célula mamária promovido pelo estradiol. Embora a progesterona não tenha influência no efeito proliferativo do estradiol na célula mamária, a testosterona pode reduzir em $40 \%$ esse efeito e abolir a expressão dos receptores $\alpha$ - estrogênicos (ER- $\alpha$ ). Vários estudos já concluíram que o andrógeno induz uma "down regulation" na proliferação epitelial mamária $\mathrm{e}$ expressão do receptor estrogênico, sugerindo que a associação estrógeno/andrógeno na terapia hormonal da menopausa possa reduzir o risco de câncer de mama (25). Não foi demonstrada relação direta entre níveis de androgênios endógenos e libido. A resposta ocorreu apenas com doses suprafisiológicas de testosterona, cuja segurança em longo prazo é incerta. Na mulher o excesso de androgênios pode levar a repercussões estéticas como acne, hirsutismo e até virilização. Podem ocorrer agressividade, retenção hídrica e aumento da pressão arterial. Laboratorialmente há tendência a policitemia, diminuição de HDL e aumento do fibrinogênio. Os androgênios aumentam a gordura visceral, os ácidos graxos livres e prejudicam a ação da insulina. Pode ocorrer dano hepático com as formulações por via oral. São várias as apresentações para o uso de testosterona na mulher. De forma geral, a testosterona injetável não é recomendada devido à natureza farmacológica dessa via acarretar variações importantes nos níveis circulantes bem como o depósito do esteroide. Por outro lado, a via intramuscular tem se mostrado eficiente em mulheres ooforectomizadas. A metiltestosterona em baixas doses $(1,25-2,5 \mathrm{mg})$ tem se mostrado eficiente no alívio dos sintomas menopausais, massa óssea, função sexual e variáveis de qualidade de vida. Undecanoato de testosterona via oral está disponível na Europa e no Canadá e é de absorção preferencialmente linfática. Os implantes de testosterona são inseridos a cada 4 a 6 meses de intervalo, a monitorização dos níveis circulantes é fundamental para a segurança da paciente e jamais devem exceder os níveis fisiológicos (70-90 ng/dL). Os adesivos de testosterona para mulher ainda não são comercializados no Brasil, mas os estudos que usaram de 150 a $300 \mu \mathrm{g}$ mostraram-se bastante satisfatórios. O gel hidroalcoólico de testosterona usado para mulheres ( $\mathrm{lg} /$ dia) na dose de $1 / 5$ do valor utilizado em homens pode ser eficiente para a composição corporal, força muscular e função sexual. O DHEA, embora usado em vários estudos na dose de $50 \mathrm{mg} /$ dia, tem sua única eficácia liberando testosterona. Até o presente não está recomendado para THM em mulheres com função adrenal preservada (26).

\section{TERAPIAS ALTERNATIVAS}

\section{Tibolona}

Tibolona é um esteroide sintético aprovado em 90 países para tratar os sintomas menopausais, incluindo Europa e Austrália mas não nos Estados Unidos e, em 45 países, está aprovado para prevenção de osteoporo- 
se. Esse composto é metabolizado em compostos com atividade estrogênica ( $3 \alpha$ e $3 \beta$ ) que, por sua vez, são convertidos no isômero $\Delta 4$, o qual tem afinidade com os receptores de progesterona e androgênio. A tibolona também diminui os níveis circulantes de SHBG aumentando a testosterona livre e contribuindo para a androgenicidade da droga. A tibolona alivia os sintomas vasomotores, melhora a atrofia urogenital, previne a perda de massa óssea e acarreta aumento da densidade óssea. Devido seu perfil androgênico, pode melhorar a libido e elevar os níveis de LDL circulantes. Prescreve-se a tibolona de forma contínua acarretando atrofia endometrial com consequente amenorreia $(7,14)$.

\section{Raloxifeno}

O SERM raloxifeno exerce efeitos estrogênicos no osso e lípides e antiestrogênicos na mama, no útero, no epitélio vaginal e em centros cerebrais promotores dos fogachos. Como resultado, na dose de $60 \mathrm{mg} / \mathrm{dia}$ melhora a densidade mineral óssea e reduz a incidência de fratura vertebral, mas não reduz a não vertebral. À semelhança do estrógeno, aumenta o risco de acidente vascular cerebral e de tromboembolismo. Como resultado de suas ações antiestrogênicas, o raloxifeno reduz a incidência de câncer de mama e endométrio, entretanto piora os sintomas vasomotores.

\section{Hormônios bioidênticos}

Não existem razões médicas ou científicas para recomendar "hormônios bioidênticos" não registrados. As preparações hormonais "customizadas" não foram testadas em estudos e sua pureza e riscos são desconhecidos (16).

\section{CONTRAINDICAÇÕES ABSOLUTAS À ESTROGENOTERAPIA}

Em função das diferentes doses e vias de administração, atualmente as contraindicações são muito poucas, entretanto ainda são considerados como contraindicações câncer de mama, câncer de endométrio, tromboembolismo agudo, hepatopatia aguda e/ou grave, cardiopatia grave e sangramento uterino sem causa diagnosticada.

\section{QUANDO COMEÇAR E QUANDO PARAR A THM}

A análise adicional dos estudos prospectivos randomizados controlados indica que a THM deve ser adminis- trada precocemente, na perimenopausa, em um grupo alvo entre 50 e 59 anos de idade (22). Nessa população a THM pode conferir proteção cardiovascular, ao passo que o início em idade avançada, após 10 anos sem estrógeno endógeno, pode ser prejudicial. A duração da THM é um dos maiores desafios do tema analisado e os dados atuais são inconsistentes para definir quando interromper a hormonioterapia. Para a reposição com estrógeno isolado, existe maior flexibilidade quanto ao tempo de uso. A decisão de manter a reposição deve ser individualizada com base nos sintomas e monitorizada e mantida enquanto os benefícios forem superiores aos riscos sempre sob supervisão médica.

Aguardamos que, em um futuro próximo, novas moléculas com efeitos máximos e riscos mínimos sejam descobertas e incluídas na THM. A genotipagem individual identificará a paciente que possui risco real para câncer de mama, tromboembolismo ou evento cardiovascular. Enquanto isso não nos deixemos levar pela mídia leiga, estudos sem o rigor científico necessário para levarmos em conta suas conclusões e utilizemos as informações atuais, cientificamente relevantes. A THM continua sendo a terapêutica de escolha para os sintomas menopausais e sua segurança depende da boa indicação, monitorização e individualização. Mulheres acima dos 60 anos de idade não devem iniciar a THM. Os consensos atuais são unânimes quando relatam que os benefícios da THM são máximos quando iniciada na perimenopausa. A mulher de 50 a 59 anos ou com menos de 10 anos de menopausa é o grupo alvo e representa a faixa de mulheres na qual os benefícios na grande maioria das vezes superam os riscos.

Declaração: os autores declaram não haver conflitos de interesse científico neste estudo.

\section{REFERÊNCIAS}

1. Rozenberg S, Vandromme J, Antoine C. Postmenopausal hormone therapy: risks and benefits. Nat Rev Endocrinol. 2013;9,216-27.

2. Sprague BL, Trentham-Dietz A, Cronin KA. A sustained decline in postmenopausal hormone use: results from the National Health and Nutrition Examination Survey, 1999-2010. Obstet Gynecol. 2012;120:595-603.

3. Hulley S, Grady D, Bush T, Furberg C, Herrington D, Riggs B, et al. Randomized trial of estrogen plus progestin for secondary prevention of coronary heart disease in postmenopausal women. Heart and Estrogen/progestin Replacement Study (HERS) Research Group. JAMA. 1998;280:605-13.

4. Rossouw JE, Anderson GL, Prentice RL, LaCroix AZ, Kooperberg C, Stefanick ML, et al.; Writing Group for the Women's Health Initiative Investigators. Risks and benefits of estrogen plus progestin in healthy postmenopausal women: principal results 
From the Women's Health Initiative randomized controlled trial. JAMA. 2002;288:321-33.

5. Anderson GL, Limacher M, Assaf AR, Bassford T, Beresford SA, Black $\mathrm{H}$, et al.; Women's Health Initiative Steering Committee. Effects of conjugated equine estrogen in postmenopausal women with hysterectomy: the Women's Health Initiative randomized controlled trial. JAMA. 2004;291:1701-12.

6. Levels of evidence and grades of recommendations - Oxford Centre for Evidence-Based Medicine. Disponível em: URL:http:// cebm.jr2.ox.ac.uk/docs/old_levels.html

7. Santen RJ, Allred DC, Ardoin SP, Archer DF, Boyd N, Braunstein $\mathrm{GD}$, et al.Postmenopausal hormone therapy: an Endocrine Society scientific statement. J Clin Endocrinol Metab. 2010,95(7 Suppl 1):S1-S66.

8. Maclennan AH, Broadbent JL, Lester S, Moore V. Oral oestrogen and combined oestrogen/progestogen therapy versus placebo for hot flushes. Cochrane Database Syst Rev. 2004;18(4):CD002978.

9. Hall E, Frey BN, Soares CN. Non-hormonal treatment strategies for vasomotor symptoms. Drugs. 2011;71(3):287-304.

10. Mochrer B, Hextall A, Jackson S. Oestrogens for urinary incontinence in women. Cochrane Database Syst Rev. 2003;2:CD001405.

11. Management of symptomatic vulvovaginal atrophy: 2013 position statement of The North American Menopause Society. Menopause. 2013:20(9):888-902.

12. Management of osteoporosis in postmenopausal women: 2010 position statement of The North American Menopause Society. Menopause. 2010;17(1):25-54.

13. Sare GM, Gray LJ, Bath PM. Association between hormone replacement therapy and subsequent arterial and venous vascular events: a meta-analysis. Eur Heart J. 2008:29:2031-41.

14. Lobo RA. Where are we 10 years after the Women's Health Initiative? J Clin Endocrinol Metab. 2013;98:1771-80.

15. Pardini DP. Terapia de reposição hormonal na menopausa. Endocrinologia Clínica.Lucio Vilar. 5.ed. Rio de Janeiro: Guanabara Koogan; 2013. p. 49.

16. Writing Group on behalf of Workshop Consensus Group Aging, menopause, cardiovascular disease and HRT. International Menopause Society Consensus Statement. Climateric. 2009;12:368-77.
17. Schierbeck LL, Rejnmark L, Tofteng CL, Stilgren L, Eiken $P$, Mosekilde $L$, et al. Effect of hormone replacement therapy on cardiovascular events in recently postmenopausal women: randomized trial. BMJ. 2012;345.

18. Kongnyuy E, Norman RJ, Flight IHK, Rees MC. 2010. Oestrogen and progestogen hormone replacement therapy for perimenopausal and post-menopausal women: weight and body fat distribution. Intervention Review: Issue 11.

19. Davis SR, Castelo-Branco C, Chedraui P, Lumsden MA, Nappi $\mathrm{RE}$, Shah D, et al.; Writing Group of the International Menopause Society for World Menopause Day 2012. Understanding weight gain at menopause. Climateric. 2012;15:419-29.

20. Salpeter SR, Cheng J, Thabane L, Buckley NS, Salpeter EE. Bayesian meta-analysis of hormone therapy and mortality in younger postmenopausal women. Am J Med. 2009;122:1016-22.

21. Sitruk-Ware R. Therapeutic use of progestins: practical recommendations In: Sitruck-Ware R, et al, eds. Progestins and antiprogestins in clinical practice. New York: M. Dekker, 2000. p. 341-53.

22. North American Menopause Society. The 2012 hormone therapy position paper of the North American Menopause Society. Menopause. 2012;19:257-71.

23. Braunstein GD. Androgen insufficiency in women: summary of critical issues. Fertil Steril. 2002;77 Suppl 4:S94-9.

24. Davison SL, Bell R, Donath S, Montalto JG, Davis SR. Androgen levels in adult females: changes with age, menopause, and oophorectomy. J Clin Endocrinol Metab. 2005;90(7):3847-53.

25. Miller KK, Biller BM, Beauregard C, Lipman JG, Jones J, Schoenfeld D, et al. Effects of testosterone replacement in androgen-deficient women with hypopituitarism: a randomized, double-blind, placebo-controlled study. J Clin Endocrinol Metab. 2006;91:1683-90.

26. Davis SR, Panjari M, Stanczyk FZ. Clinical review: DHEA replacement for postmenopausal women. J Clin Endocrinol Metab. 2011;96(6):1642-53.

27. Birkhäuser $M H$, Panay $N$, Archer DF, Barlow $D$, Burger $H$, Gambacciani M, et al. Updated practical recommendations for hormone replacement therapy in the peri- and postmenopause. Climacteric. 2008;11:108-23. 